\title{
Atypical Presentation of a Maxillary Chondroblastic Osteosarcoma and Complex Management
}

\author{
Jayan George 1,*®D, Mohamed Abdulla ${ }^{2}$, Amir Farboud ${ }^{2}$, Gareth Leopold ${ }^{3}$ and Conor Marnane ${ }^{2}$ \\ 1 General Surgical Department, Sheffield Teaching Hospitals NHS Foundation Trust, Herries Road, \\ Sheffield S5 7AU, UK \\ 2 ENT Head and Neck surgery department, Morrison Hospital, Swansea SA6 6NL, UK; \\ mohamed.r.abdulla@gmail.com (M.A.); amirfarboud@doctors.net.uk (A.F.); \\ conor.marnane@wales.nhs.uk (C.M.) \\ 3 Histopathology department, Morrison Hospital, Swansea SA6 6NL, UK; gareth.leopold@wales.nhs.uk \\ * Correspondence: jayan.george@aol.com; Tel.: +44-1142-434-343; Fax: +44-1142-266-986
}

Received: 16 October 2018; Accepted: 13 November 2018; Published: 15 November 2018

check for updates

\begin{abstract}
We report a rare case of a 23-year-old male who presented with a four-week history of unilateral recurrent epistaxis and nasal obstruction. This was subsequently diagnosed as high-grade chondroblastic osteosarcoma of the maxillary antrum. Neo-adjuvant chemotherapy was commenced following a multidisciplinary team discussion. The disease progressed through first-line treatment. A radical surgical resection was not possible, and a palliative debulking procedure was performed. The literature comprehensively points towards an early surgical resection with suitable margins at the earliest possible stage. This case highlights that osteosarcoma of the maxilla can be challenging to diagnose and manage. Thus, a high index of suspicion and an early referral to a head and neck specialist is imperative to improve the long-term prognosis in such patients.
\end{abstract}

Keywords: chondrosarcoma; maxilla osteosarcoma; epistaxis

\section{Introduction}

A sarcoma is a malignant mesenchymal tumour associated with varied clinical presentations and pathological classifications. In the craniofacial region rhabdomyosarcoma or osteosarcoma (OS) are most commonly seen [1]. OS makes up approximately $20 \%$ of all sarcomas and is the most common non-haematopoietic primary bone neoplasm, with a reported incidence of around 1 in 100,000 [2]. Involvement of the craniofacial skeleton remains relatively rare, with an estimated incidence of less than 1 per million and is found only in $6-10 \%$ of all OS cases [3]. Long bone OS and craniofacial OS share microscopic features; however, they are considered as separate entities [4]. OS of the long bones age of onset tends to be a bimodal distribution from adolescence and the fifth decade, the most common cause of mortality is distant metastasis, painful swelling as well as the rarity of neuroparaesthesia amongst other factors $[5,6]$. OS of the jaw bones differ as most occur during the third or fourth decade, locoregional recurrence is tbhe most common cause of mortality; painless swelling is normally the first sign and neuroparaesthesia is normally present $[5,7,8]$.

We report the case of a 23-year-old male with a four-week history of recurrent epistaxis and unilateral nasal obstruction. This was subsequently diagnosed as high-grade chondroblastic OS of the maxillary antrum. Due to evidence of an already locally advanced lesion, treatment using neo-adjuvant chemotherapy was recommended. The patient underwent first and second-line treatment, which unfortunately failed to slow down the rate of progression. We highlight the challenges faced by clinicians in achieving a timely and accurate histopathological diagnosis and summarise the current evidence in the management of such tumours. 


\section{Case Presentation Section}

A 23-year-old male university student of South East Asian origin presented to our Ear, Nose and Throat department with a four-week history of increased frequency epistaxis and unilateral obstruction of the right nasal cavity. Seven days prior to his admission he had been treated for a presumed maxillary sinusitis by the general practitioner (GP).

The episodes of epistaxis were usually mild and self-limiting after a period of localised pressure. He described increasing pain and fullness over his cheek and nasal cavity. Apart from symptoms of lethargy, he was otherwise well with no significant past medical history. He had been previously treated for episodes of mild recurrent allergic rhinitis. There was no family history of cancer or predisposing genetic condition. He was a non-smoker and drank occasionally.

On examination, a $1 \mathrm{~cm}$ friable, pigmented lesion obstructing the right nasal cavity was visible. The rest of his head and neck examination was unremarkable and there was no evidence of cervical lymphadenopathy.

A biopsy of the lesion showed cartilage with overlying inflamed granulation tissue, with no evidence of any malignancy seen. Blood results, including a vasculitic screen were unremarkable. Magnetic resonance angiogram (MRI) and computed tomographic (CT) scan of the nasal sinuses showed a calcified osteoid sinonasal mass obstructing the right posterior nasal cavity. It appeared chondroid in nature, arising from the medial wall of the right maxillary antrum (Figure 1).

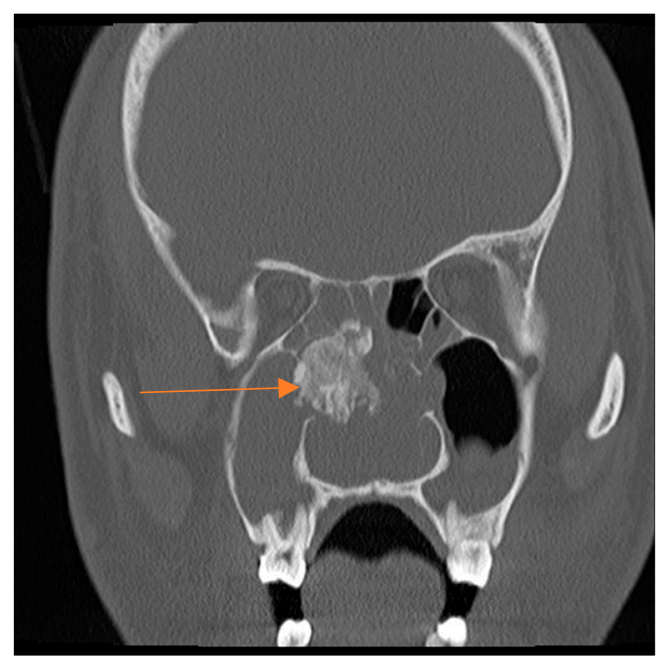

Figure 1. Coronal computed tomographic (CT) showing a calcified sinonasal mass (orange arrow), which is chondroid in nature arising from the medial wall of the right maxillary antrum.

The head and neck cancer multidisciplinary team (MDT) meeting decided that an urgent nasal polypectomy was warranted. This revealed a possible diagnosis of grade III chondrosarcoma based on mitotic rate. A second opinion felt that such findings were more in keeping with high-grade chondroblastic OS and this was discussed formally in the MDT (Figures 2 and 3). 


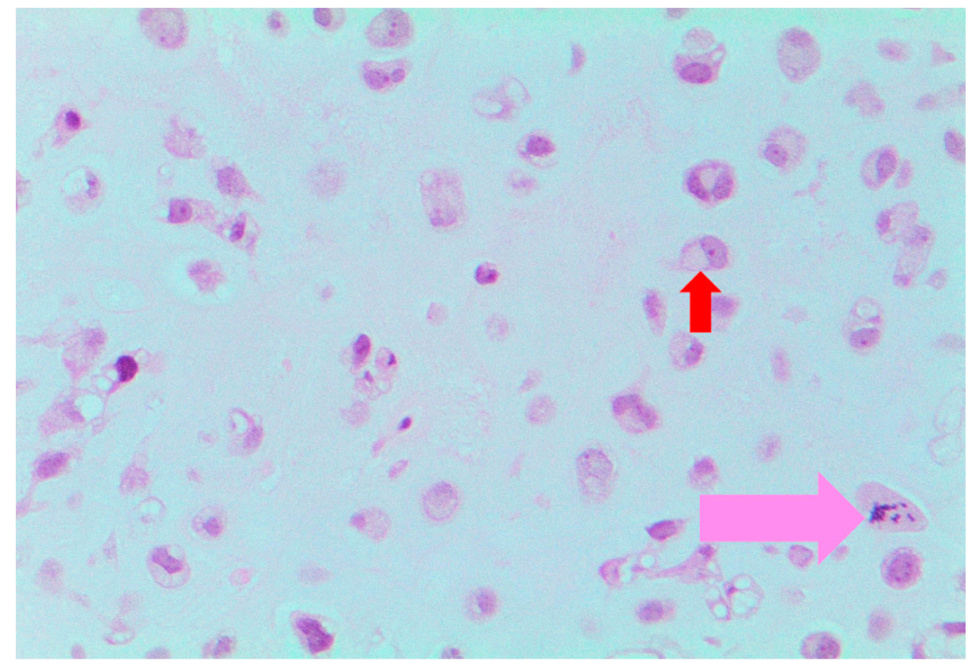

Figure 2. Histopathology slide $\times 20$ showing the chondroblastic component of the tumour; chondroblasts (red arrow); mitotic figure (pink arrow).

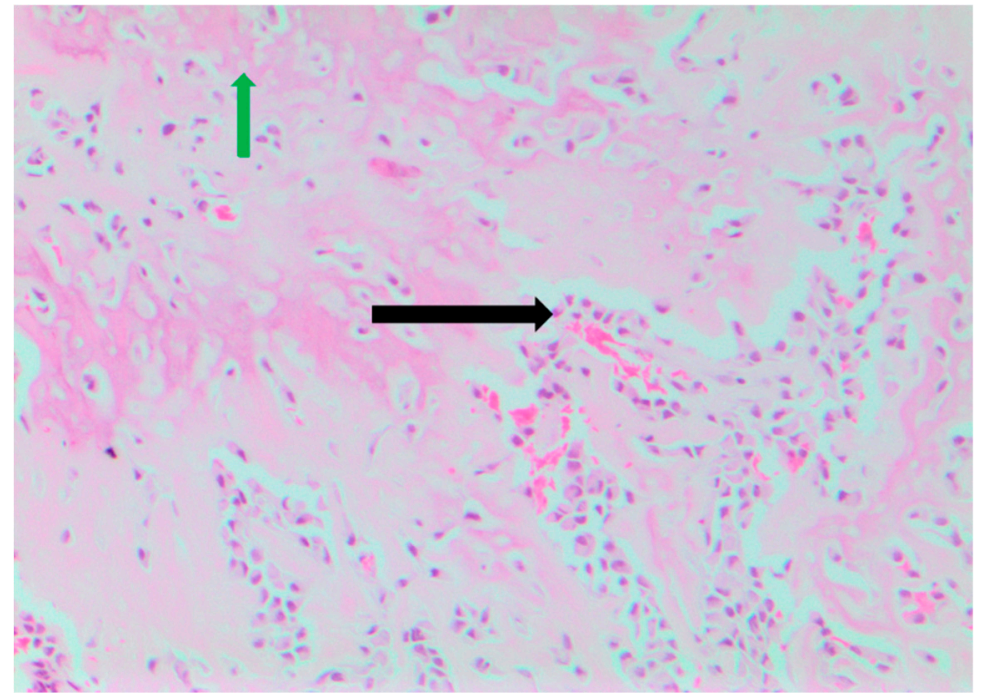

Figure 3. Histopathology slide $\times 10$ showing the osteosarcomatous component of the tumour; osteoblasts (black arrow), and osteoid/new bone formation (green arrow).

The national sarcoma MDT recommended neo-adjuvant chemotherapy for a period of ten weeks followed by surgery. The decision was made to treat as per the European and American Osteosarcoma study group trial 1 protocol (EURAMOS-1) [9]. As outlined by the protocol, the patient began first-line chemotherapy using a combination of Cisplatin, Doxorubicin and high dose Methotrexate.

A CT scan following the first cycle of treatment showed an extension of the tumour towards the oropharynx. There was evidence of erosion into the right orbital cavity and posterior wall of the right maxillary antrum into the masticator space.

The tumour was visible externally, disfiguring for the patient and given the location there was a high risk of airway compromise and he underwent tracheostomy. Radiologically inserted gastrostomy was sited. Palliative debulking surgery was carried out to improve patient function and to achieve local control.

The patient developed neutropenic sepsis and became dehydrated and developed an acute kidney injury. He also experienced grade 2 mucositis, which was managed supportively. 
An MRI after the completion of first-line chemotherapy demonstrated a progressive disease. There was evidence of increased expansion superiorly into the orbital cavity up to the optic nerve sheath complex and inferiorly through the hard palate, filling of most of the pharynx (Figures 4 and 5).



Figure 4. Coronal Magnetic resonance angiogram (MRI) showing distension of the right nasal cavity (orange arrow) and extension into the pterygopalatine fossa and pterygoid process of the sphenoid bone indicative of a malignant pathology (yellow arrow).

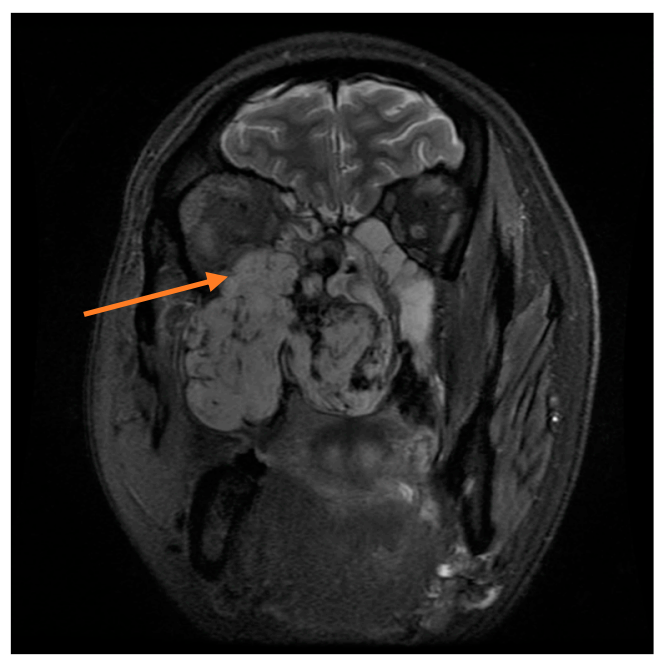

Figure 5. Coronal MRI demonstrating expansion superiorly into the right orbital cavity (orange arrow).

The patient started second-line chemotherapy with Ifosfamide and Etoposide. A CT scan following second-line treatment showed further local invasion by the tumour. There was no evidence of complete occupation of the pharynx down to the level of the glottis (Figure 6).

The MDT formal outcome advised palliative radiotherapy to a dose of 20 Gy in 5 fractions was given after the patient developed significant epistaxis. The patient was repatriated back to his home country during his palliative treatment.

Ethics and Consent: Verbal consent was gained directly from the patient before death. Efforts were made to make contact with the patients next of kin however this was unsuccessful. As such, no patient identifiable data is present in this report. 


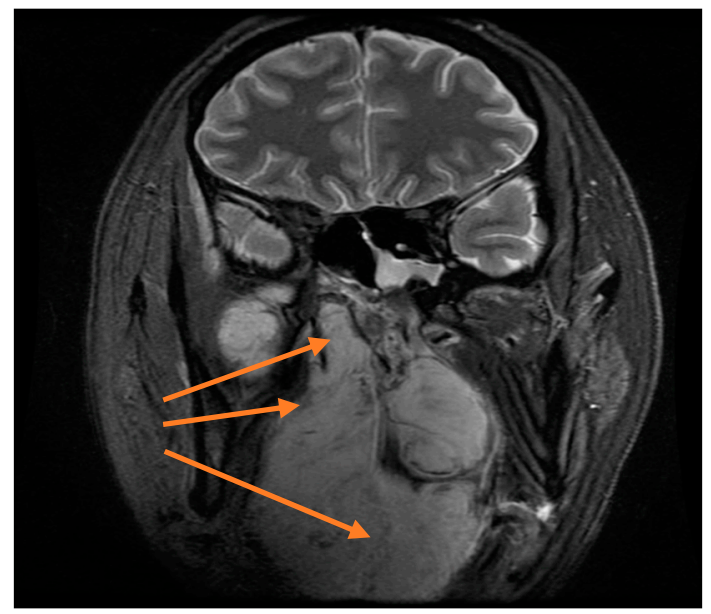

Figure 6. Coronal MRI showing extension into the naso and oropharynx (orange arrows).

\section{Discussion}

OS is histologically characterised by the presence of malignant and undifferentiated cellular stroma that produce neoplastic osteoid or primitive bone [1,4]. The histological subtype of OS is classified according to the predominant matrix material present, depending on whether it is osteoid (osteoblastic), cartilaginous (chondroblastic) or minimal matrix (fibroblastic) [10]. The predominant matrix was that of cartilaginous tissue in this patient.

It is essential that chondroblastic OS is differentiated from chondrosarcoma, as the latter is associated with a better prognosis and rarely occurs in adolescents or young adults $[4,11]$. Due to the presence of cartilaginous tissue, small specimens can mislead pathologists into diagnosing a chondrosarcoma. This is due to the lack of osteoid production that is visible during analysis when a specimen is obtained using techniques, such as incisional biopsies [4,12]. The false diagnosis rate is believed to be as high as $44 \%$ [13].

Immunohistochemistry plays a vital role in helping to differentiate between both these diagnoses. Both conditions are known to be positive for Vimentin and S100; however, chondroblastic OS displays positivity for Epithelial Membrane Antigen (EMA) and Cytokeratin [13]. It is believed the average time taken to establish a histopathological diagnosis from the onset of symptoms is between three to four months [4]. Our patient was diagnosed approximately two and a half months from the onset of his symptoms. Following a second opinion from the national sarcoma team, a high-grade chondroblastic OS was favoured over a chondrosarcoma with the aid of immunohistochemistry.

The early clinical presentation of craniofacial OS is usually non-specific and can delay the referral to a specialist [1]. August et al found that a painless swelling was the most common presentation amongst a series of thirty patients [7]. Other common signs and symptoms included paraesthesia, loosening or displacement of teeth or dentures, headaches [7,14]. A recent case series reviewed 47 patients with osteosarcomas of the jaw and found the average age for males was 38.5 years, epistaxis accounted for $4 \%$ of cases and $97.8 \%$ were Caucasian [14]. Literature suggests that environmental factors combined with genetic mutations could provide a possible explanation in such patients [15]. The patient presented with a history of recurrent unilateral epistaxis and nasal obstruction, this combined with his age and unremarkable past medical and family history is quite unique for this condition.

These are, however, red flag signs associated with malignancy during the patient's last visit to the GP, where he was treated for a suspected acute sinusitis. This was a combination of his symptoms (unilateral recurrent epistaxis, nasal obstruction and increasing facial pain) and ethnic origin [16].

In the maxilla, the most commonly affected sites involve the posterior alveolar process and the antrum. The site of origin in this patient was the medial wall of the right maxillary antrum. Due to the nature of the tumour, which invades cranially rather than caudally, patients often present at a stage 
where local invasion has already occurred [7]. This could be why symptoms such as nasal obstruction, epistaxis or masticatory problems are often poor prognostic signs [14].

$\mathrm{CT}$ and MRI scans are the most commonly used form of imaging in craniofacial OS. They are often preferred due to their ability to detect intra- and extra-medullary involvement and invasion into structures such as the skull base and the infratemporal fossa [17]. The patient received regular follow-up via MRI and CT scans to assess his response to treatment. Following treatment with second-line chemotherapy, his concluding CT scan showed evidence of increased local invasion into the base of the skull, oral cavity and pharynx when compared with previous imaging.

The mainstay of treatment and the single most important prognostic factor of craniofacial OS is surgical resection with an optimal clearance margin of 15-20 $\mathrm{mm}[15,18]$. An adequate clearance during a maxillectomy can prove to be difficult to accomplish, particularly when the tumour has already begun to invade and involve structures in the pterygopalatine and infratemporal fossa. The incidence of positive margins associated with resections of the maxilla or skull base has been reported to be in the range of $30-54.5 \%$ of all cases [18]. Given the patient's advancing condition and the psychological effect of having such a disfigurement and airway compromise, palliative debulking surgery was the only option to improve the patient's quality of life.

The use of neoadjuvant chemotherapy has been used widely in craniofacial OS, a significant improvement in survival rates has been demonstrated in case series [19].

High-grade chondroblastic OS of the maxilla can be very challenging to diagnose and manage. This patient's presentation coupled with his age, ethnicity, past medical and family history makes this diagnosis extremely rare but a high index of suspicion and an early referral to a head and neck specialist is essential. Histopathological diagnosis is challenging and takes time. Surgical resection is the mainstay of treatment if acceptable margins can be achieved. In order to obtain a timely histopathological diagnosis and allow a potentially curative approach to treatment.

Author Contributions: J.G.: Developing and proofing the manuscript and editing the images. M.A.: Developing and proofing the manuscript. A.F.: Developing and proofing the manuscript. G.L.: Attaining, annotating the histopathology slides and proofing the manuscript. C.M.: Developing and proofing the manuscript.

Funding: This research received no external funding.

Acknowledgments: The authors would like to thank Samuel Birks for editing the images.

Conflicts of Interest: The authors declare no conflict of interest.

\section{References}

1. Santos-Silva, A.R.; Ribeiro, A.C.P.; Furuse, C.F.; Simonato, L.E.; Mattar, N.J.; Soubhia, A.M.; Miyahara, G.I. Maxillary osteosarcoma in a young patient undergoing postorthodontic treatment follow-up: The importance of ongoing oral examinations. Am. J. Orthod. Dentofacial Orthop. 2011, 139, 845-848. [CrossRef] [PubMed]

2. Kassir, R.R.; Rassekh, C.H.; Kinsella, J.B.; Segas, J.; Carrau, R.L.; Hokanson, J.A. Osteosarcoma of the head and neck: Meta-analysis of nonrandomized studies. Laryngoscope 1997, 107, 56-61. [CrossRef] [PubMed]

3. Cutilli, T.; Scarsella, S.; Fabio, D.; Oliva, A.; Cargini, P. High-grade chondroblastic and fibroblastic osteosarcoma of the upper jaw. Ann. Maxillofac. Surg. 2011, 1, 176. [CrossRef] [PubMed]

4. Kumaravelu, C.; Sathya Kumar, D.; Chakravarthy, C.; Kishore Kumar, R.V.; Rajasekhar, G.; Gokul Chandra Reddy, M.; Sridhar Reddy, K. Chondroblastic osteosarcoma of maxilla: A case report and review of literature. J. Maxillofac. Oral Surg. 2009, 8, 290-293. [CrossRef] [PubMed]

5. Mardinger, O.; Givol, N.; Talmi, Y.P.; Taicher, S. Osteosarcoma of the jaw. The Chaim Sheba Medical Center experience. Oral Surg. Oral Med. Oral Pathol. Oral Radiol. Endod. 2001, 91, 445-451. [CrossRef] [PubMed]

6. Huvos, A.G. Osteogenic sarcoma. In Bone Tumors: Diagnosis, Treatment and Prognosis, 2nd ed.; W.B.Saunders: Philadelphia, PA, USA, 1991; pp. 85-116.

7. August, M.; Magennis, P.; Dewitt, D. Osteogenic sarcoma of the jaws: Factors influencing prognosis. Int. J. Oral Maxillofac. Surg. 1997, 26, 198-204. [CrossRef]

8. Slootweg, P.J.; Müller, H. Osteosarcoma of the jaw bones. Analysis of 18 cases. J. Maxillofac. Surg. 1985, 13, 158-166. [CrossRef] 
9. Bielack, S.S.; Smeland, S.; Whelan, J.S.; Marina, N.; Jovic, G.; Hook, J.M.; Krailo, M.D.; Gebhardt, M.; Pápai, Z.; Meyer, J.; et al. Methotrexate, Doxorubicin, and Cisplatin (MAP) Plus Maintenance Pegylated Interferon Alfa-2b Versus MAP Alone in Patients With Resectable High-Grade Osteosarcoma and Good Histologic Response to Preoperative MAP: First Results of the EURAMOS-1 Good Respons. J. Clin. Oncol. 2015, 33, 2279-2287. [CrossRef] [PubMed]

10. Padilla, R.J.; Murrah, V.A. The Spectrum of Gnathic Osteosarcoma: Caveats for the Clinician and the Pathologist. Head Neck Pathol. 2011, 5, 92-99. [CrossRef] [PubMed]

11. Vercruysse, J.P.; Claes, J. Chondrosarcoma of the nasal septum: A report of two cases. B-ENT 2006, 2, 27-30. [PubMed]

12. Coca-Pelaz, A.; Rodrigo, J.P.; Triantafyllou, A.; Hunt, J.L.; Fernández-Miranda, J.C.; Strojan, P.; de Bree, R.; Rinaldo, A.; Takes, R.P.; Ferlito, A. Chondrosarcomas of the head and neck. Eur. Arch. Otorhinolaryngol. 2014, 271, 2601-2609. [CrossRef] [PubMed]

13. Akpolat, N.; Yildirim, H.; Poyraz, K. Sacral Chondroblastic Osteosarcoma Misdiagnosed as Chondrosarcoma. Turk. J. Med. Sci. 2007, 37, 243-249.

14. LeCornu, M.G.; Chuang, S.-K.; Kaban, L.B.; August, M. Osteosarcoma of the Jaws: Factors Influencing Prognosis. J. Oral Maxillofac. Surg. 2011, 69, 2368-2375. [CrossRef] [PubMed]

15. Chaudhary, M.; Chaudhary, S.D. Osteosarcoma of jaws. J. Oral Maxillofac. Pathol. 2012, 16, $233-238$. [CrossRef] [PubMed]

16. National Institute of Clinical Excellence (NICE). Epistaxis (Nosebleeds)—Recurrent Epistaxis. Clinical Knowledge Summaries 2015. Available online: https:/ / cks.nice.org.uk/epistaxis-nosebleeds\#!scenario:1 (accessed on 22 March 2017).

17. Doval, D.C.; Kumar, R.V.; Kannan, V.; Sabitha, K.S.; Misra, S.; Vijay Kumar, M.; Hegde, P.; Bapsy, P.P.; Mani, K.; Shenoy, A.M.; et al. Osteosarcoma of the jaw bones. Br. J. Oral Maxillofac. Surg. 1997, 35, 357-362. [CrossRef]

18. Fernandes, R.; Nikitakis, N.G.; Pazoki, A.; Ord, R.A. Osteogenic sarcoma of the jaw: A 10-year experience. J. Oral Maxillofac. Surg. 2007, 65, 1286-1291. [CrossRef] [PubMed]

19. Mücke, T.; Mitchell, D.A.; Tannapfel, A.; Wolff, K.-D.; Loeffelbein, D.J.; Kanatas, A. Effect of neoadjuvant treatment in the management of osteosarcomas of the head and neck. J. Cancer Res. Clin. Oncol. 2014, 140, 127-131. [CrossRef] [PubMed] 